\title{
GENERALIZED LEFSCHETZ NUMBERS OF PUSHOUT MAPS DEFINED ON NON-CONNECTED SPACES
}

\author{
DAVIDE L. FERRARIO \\ Dipartimento di Matematica, Università di Milano \\ Via Saldini 50, 20133 Milano, Italy \\ E-mail: ferrario@vmimat.mat.unimi.it
}

\begin{abstract}
Let $A, X_{1}$ and $X_{2}$ be topological spaces and let $i_{1}: A \rightarrow X_{1}, i_{2}: A \rightarrow X_{2}$ be continuous maps. For all self-maps $f_{A}: A \rightarrow A, f_{1}: X_{1} \rightarrow X_{1}$ and $f_{2}: X_{2} \rightarrow X_{2}$ such that $f_{1} i_{1}=i_{1} f_{A}$ and $f_{2} i_{2}=i_{2} f_{A}$ there exists a pushout map $f$ defined on the pushout space $X_{1} \sqcup_{A} X_{2}$. In [F] we proved a formula relating the generalized Lefschetz numbers of $f, f_{A}$, $f_{1}$ and $f_{2}$. We had to assume all the spaces involved were connected because in the original definition of the generalized Lefschetz number given by Husseini in $[\mathrm{H}]$ the space was assumed to be connected. So, to extend the result of $[\mathrm{F}]$ to the not necessarily connected case, a definition of generalized Lefschetz number for a map defined on a not necessarily connected space is given; it reduces to the original one when the space is connected and it is still a trace-like quantity. It allows us to prove the pushout formula in this more general case and therefore to get a tool for computing Nielsen and generalized Lefschetz numbers in a wide class of spaces.
\end{abstract}

1. Introduction. As explained in the abstract, the aim of the paper is to give a proof of the pushout formula in the more general case where spaces are allowed to be non-connected. The main difference between the connected and the non-connected case is that if $X$ is connected then so is the universal cover $\tilde{X}$ of $X$ and the $q$-dimensional cellular chain group $C_{q}(\tilde{X})$ is a free finitely generated right $\mathbf{Z} \pi_{1}(X)$-module; on the other hand if $X$ is disconnected then $\mathbf{Z} \pi_{1}(X ; x)$ depends on the choice of the base point $x$ and for all $x \in X$ the chain group $C_{q}(\tilde{X})$ is not a free $\mathbf{Z} \pi_{1}(X ; x)$-module. Moreover we want to have a trace of the homomorphism $C_{q}(\tilde{f})$ where $\tilde{f}: \tilde{X} \rightarrow \tilde{X}$ and so a generalized Lefschetz number counting algebraically the number of fixed points of $f$. Hence we define the ring $\Lambda(X)$ which contains $\mathbf{Z} \pi_{1}\left(X ; x_{0}\right)$ for every $x \in X$ and we prove that $C_{q}(\tilde{X})$ is a finitely generated projective $\Lambda(X)$-module. It is a free $\Lambda(X)$-module if and only if $X$ is connected. In any case, it is possible to define traces following $[\mathrm{S}],[\mathrm{H}]$ and the generalized Lefschetz numbers for non-connected spaces.

1991 Mathematics Subject Classification: Primary 55M20, Secondary 55P99.

The paper is in final form and no version of it will be published elsewhere. 
We first have to extend the notion of Reidemeister classes to the case of a ring which could be not a group ring. This is done in section 2.1; in the same section we prove other propositions which will be used later. In the following sections we prove standard properties of traces and Lefschetz numbers in this algebraic setting. In section 3.1 we define the generalized Lefschetz number of a map defined on a finite $C W$-complex even when $X$ is not connected and give the relation between this case and the connected one.

In section 3.2 we give standard definitions and examples of pushout construction and some preliminary facts. Finally in section 4 we give the statement and proof of the pushout formula.

I wish to express my sincere thanks to the Organizing Committee and in particular to Prof. Brown and Prof. Kucharski. I wish to thank Prof. Piccinini for his help.

\section{Algebraic preliminaries}

2.1. The Reidemeister group of a ring homomorphism. In this section we will introduce a generalization of the classical Reidemeister set defined for group homomorphisms and will show some simple facts that will be needed later.

Let $\Lambda$ be a ring (with unit element) and $f: \Lambda \rightarrow \Lambda$ be an endomorphism of $\Lambda$. Let $(\Lambda)_{f}$ denote the subgroup of $\Lambda$ generated by all the elements $\lambda_{1} \lambda_{2}-\lambda_{2} f\left(\lambda_{1}\right)$ with $\lambda_{1}, \lambda_{2} \in \Lambda$. We define the Reidemeister group of $f$ as the additive group of $\Lambda$ modulo $(\Lambda)_{f}$ and we will denote it with $\mathcal{R}(f)$. We will denote by $[\lambda]$ the obvious projection of $\lambda$ in $\mathcal{R}(f)$.

EXAmple 1. If $f=1_{\Lambda}$ then $\mathcal{R}(f)$ is the group defined in [S], page 130 .

EXAMPLE 2. If $\Lambda=\mathbf{Z} G$ is the group ring of a group $G$ over the ring of integers $\mathbf{Z}$ and $f=\mathbf{Z} \varphi$ is the linear extension of a group endomorphism $\varphi: G \rightarrow G$ then $\mathcal{R}(f)$ is the free abelian group generated by the set $R(\varphi)$ of orbits in $G$ of the action of $G$ over $G$ defined by $g \cdot x:=g x \varphi\left(g^{-1}\right)(\forall g, x \in G)$. In other words it is the classical Reidemeister set of a group homomorphism (see e.g. [B], [FH], [H], [J]).

Proof. Let $R(\varphi)$ denote the orbit set and $[g]$ denote the orbit of $g \in G$. The context will make clear whether $[g]$ is seen as an element of $\mathcal{R}(f)$ or of $R(\varphi)$. We want to prove that $\mathcal{R}(f) \equiv \mathbf{Z} R(\varphi)$. Let $p_{0}: G \rightarrow \mathbf{Z} R(\varphi)$ be defined by $p_{0}(g)=[g]$ for each $g \in G$ and let $p$ be the linear extension of $p_{0}$ to $\mathbf{Z} G$. Because $p$ is onto, it suffices to prove that $\operatorname{Ker}(p)=(\Lambda)_{f}$. But $g_{1} g_{2}=g_{1} g_{2} f\left(g_{1}\right) f\left(g_{1}^{-1}\right)$ and hence $p\left(g_{1} g_{2}-g_{2} f\left(g_{1}\right)\right)=0 \quad\left(\forall g_{1} g_{2} \in G\right)$ therefore $\operatorname{Ker}(p) \supseteq(\Lambda)_{f}$. Now let $\lambda \in \operatorname{Ker}(p)$; this means that $\lambda=n_{1} g_{1}+n_{2} g_{2}+\ldots+n_{k} g_{k}$ with $n_{i} \in \mathbf{Z}$ and $g_{i} \in G$ for all $i=1, \ldots, k$ and that $\sum_{i=1}^{k} n_{i}\left[g_{i}\right]=0$. Up to rearranging indices, we can suppose that $\left[g_{1}\right]=\left[g_{2}\right]=\ldots=\left[g_{k_{1}}\right],\left[g_{k_{1}+1}\right]=\ldots=\left[g_{k_{2}}\right], \ldots,\left[g_{k_{l}+1}\right]=\ldots=\left[g_{k}\right]$ with $1 \leq k_{1} \leq k_{2} \leq \ldots \leq k_{l} \leq k$ suitable integers. In other words $\lambda=\sum_{j=0}^{l} \mu_{j}$ where $\mu_{j}=\sum_{i=k_{j}+1}^{k_{j+1}} n_{k_{j}+i} g_{k_{j}+i}$ and $k_{0}=0, k_{l+1}=k$. As $\mathbf{Z} R(\varphi)$ is free, $p(\lambda)=0$ implies $p\left(\mu_{j}\right)=0$ for all $j$. Therefore it is enough to prove that $\lambda \in(\Lambda)_{f}$ in the simple case $k_{1}=k$ when $\left[g_{1}\right]=\ldots=\left[g_{k}\right]$. In this case there exist elements $\xi_{2}, \ldots, \xi_{k} \in G$ such that $g_{i}=\xi_{i} g_{1} \varphi\left(\xi_{i}^{-1}\right)$ for $i=2, \ldots, k$ and $n_{1}+\sum_{i=2}^{k} n_{i}=0$. Hence

$$
\lambda=n_{1} g_{1}+n_{2} g_{2}+\ldots+n_{k} g_{k}=-\sum_{i_{2}}^{k} n_{i} g_{1}+\sum_{i=2}^{k} n_{i} \xi_{i} g_{1} \varphi\left(\xi_{i}^{-1}\right)
$$




$$
=-\sum_{i=2}^{k} n_{i}\left(\left(\xi_{i}^{-1}\right)\left(\xi_{i} g_{1}\right)-\left(\xi_{i} g_{1}\right)\left(\varphi\left(\xi_{i}^{-1}\right)\right)\right)
$$

which is an element of $(\Lambda)_{f}$.

Consider the rings $\Lambda_{1}, \Lambda_{2}$ and the following commutative diagram of ring homomorphisms:

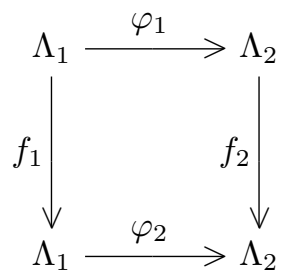

If $\Lambda_{1}=\sum_{i=1}^{p} \Lambda_{1}^{i}$ (direct sum) with $f_{1}\left(\Lambda_{1}^{i}\right) \subset \Lambda_{1}^{i}$ and $\left(\Lambda_{1}^{i}\right)_{\left.f_{1}\right|_{\Lambda_{1}^{i}}}=\left(\Lambda_{1}\right)_{f_{1}} \cap \Lambda_{1}^{i}$ for each $i=1, \ldots, p$ we will say that $\Lambda_{1}$ is well-decomposed. This implies that $\mathcal{R}\left(f_{1}\right)=$ $\sum_{i=1}^{p} \mathcal{R}\left(\left.f_{1}\right|_{\Lambda_{1}^{i}}\right)$.

Proposition 2.1. If $\Lambda_{1}$ is well-decomposed into $\sum_{i=1}^{p} \Lambda_{1}^{i}$ and for every $i=1, \ldots, p$ there exists $\theta_{i} \in \Lambda_{2}$ such that for all $\lambda_{i} \in \Lambda_{1}^{i}$

$$
\theta_{i} \varphi_{2}\left(\lambda_{i}\right)=\varphi_{1}\left(\lambda_{i}\right) \theta_{i}
$$

then there exists a well-defined group homomorphism $\theta_{*}: \mathcal{R}\left(f_{1}\right) \rightarrow \mathcal{R}\left(f_{2}\right)$ given by $\theta_{*}\left(\left[\sum_{i=1}^{p} \lambda_{i}\right]\right)=\sum_{i=1}^{p}\left[\theta_{i} \varphi_{2}\left(\lambda_{i}\right)\right]$ with $\lambda_{i} \in \Lambda_{1}^{i}$ for each $i=1, \ldots, p$.

Proof. Let us prove that $\theta_{*}$ is well defined. We must show that $\theta_{*}([\eta])=0$ for all $\eta \in\left(\Lambda_{1}\right)_{f_{1}}$. Because for $i=1, \ldots, p$ we have by hypothesis that $\left(\Lambda_{1}^{i}\right)_{\left.f_{1}\right|_{\Lambda_{1}^{i}}}=\left(\Lambda_{1}\right)_{f_{1}} \cap \Lambda_{1}^{i}$, then $\theta_{*}([\eta])=0$ for all $\eta \in\left(\Lambda_{1}\right)_{f_{1}}$ if and only if $\theta_{*}([\eta])=0$ for all $\eta \in\left(\Lambda_{1}^{i}\right)_{\left.f_{1}\right|_{\Lambda_{1}^{i}}}$ for $i=1, \ldots, p$.

Therefore for all $\lambda, \mu \in \Lambda_{1}^{i}$ the following equalities hold:

$$
\begin{aligned}
\mathcal{R}\left(f_{2}\right) \ni\left[\theta_{i} \varphi_{2}\left(\lambda \mu-\mu f_{1}(\lambda)\right)\right] & =\left[\theta_{i} \varphi_{2}(\lambda) \varphi_{2}(\mu)-\theta_{i} \varphi_{2}(\mu) f_{2}\left(\phi_{1}(\lambda)\right)\right] \\
& =\left[\theta_{i} \varphi_{2}(\lambda) \varphi_{2}(\mu)-\varphi_{1}(\lambda) \theta_{i} \varphi_{2}(\mu)\right] \\
& =\left[\theta_{i} \varphi_{2}(\lambda) \varphi_{2}(\mu)-\theta_{i} \varphi_{2}(\lambda) \varphi_{2}(\mu)\right]=[0]
\end{aligned}
$$

and hence $\theta_{*}$ is well-defined on $\mathcal{R}\left(f_{1}\right)$. By trivial arguments it can be shown that $\theta_{*}$ is a group homomorphism.

We note that if $\varphi_{1}=\varphi_{2}$ we can always define $\varphi_{*}:=\theta_{*}$ by setting $\theta=1$ on the whole $\Lambda_{1}$. In this case $\varphi_{*}\left(\left[\lambda_{1}\right]\right)=\left[\varphi_{2}\left(\lambda_{1}\right)\right]$ for all $\lambda_{1} \in \Lambda_{1}$.

Here we prove some elementary properties of Reidemeister groups.

Commutativity: If $g: \Lambda_{1} \rightarrow \Lambda_{2}$ and $f: \Lambda_{2} \rightarrow \Lambda_{1}$ are ring homomorphisms, then $g_{*}: \mathcal{R}(f g) \rightarrow \mathcal{R}(g f)$ is an isomorphism. In fact $f_{*}: \mathcal{R}(g f) \rightarrow \mathcal{R}(f g)$ is its inverse: $f_{*} g_{*}([\lambda])=[f g(\lambda)]=[\lambda] \forall \lambda \in \Lambda_{1}$ and $g_{*} f_{*}([\mu])=[\mu] \forall \mu \in \Lambda_{2}$.

Conjugacy: If $\theta$ is a unit element in the ring $\Lambda$ and $f: \Lambda \rightarrow \Lambda$ is a ring endomorphism we can define $\theta^{-1} f \theta$ as the endomorphism defined by $\theta^{-1} f \theta(\lambda):=\theta^{-1} f(\lambda) \theta$ for all $\lambda \in \Lambda$. Moreover if we define $\varphi_{1}(\lambda):=\lambda$ and $\varphi_{2}(\lambda):=\theta^{-1} \lambda \theta$ we get that $\varphi_{2} f=\theta^{-1} f \theta \varphi_{1}$ and 
$\theta \varphi_{2}(\lambda)=\varphi_{1}(\lambda) \theta \forall \lambda \in \Lambda$. Therefore $\varphi_{*}=\theta_{*}$ is a well-defined isomorphism $\varphi_{*}=\theta_{*}$ : $\mathcal{R}(f) \rightarrow \mathcal{R}\left(\theta^{-1} f \theta\right)$.

2.2. The trace. In this section we introduce some basic facts about the trace of $f$ homomorphisms defined on projective modules, following the lines of $[\mathrm{B}],[\mathrm{H}],[\mathrm{S}]$.

Let $\Lambda$ be a ring and $M_{1}, M_{2}$ be finitely generated right projective $\Lambda$-modules. An additive function $F: M_{1} \rightarrow M_{2}$ is an $f$-homomorphism of $M$ if $f$ is an endomorphism of $\Lambda$ and $F(x \lambda)=F(x) f(\lambda)$ for all $x \in M_{1}$ and $\lambda \in \Lambda$. If $M_{1}=M_{2}, F$ is an $f$-endomorphism. We want to define a trace function on the (additive) group of all such $f$-endomorphisms.

Let $\mathcal{M}_{p, q}(\Lambda)$ denote the group of all $p \times q$ matrices with entries in $\Lambda$. For any matrix $F$ let $F^{f}$ denote the matrix obtained by applying $f$ to each of the entries of $F$.

Proposition 2.2. For every integer $p$ there exists a unique group homomorphism

$$
\operatorname{Tr}_{f}: \mathcal{M}_{p, p} \rightarrow \mathcal{R}(f)
$$

such that

$$
\operatorname{Tr}_{f}(F G)=\operatorname{Tr}_{f}\left(G F^{f}\right)
$$

for all $F \in \mathcal{M}_{q, p}$ and $G \in \mathcal{M}_{p, q}$. It is given by $\operatorname{Tr}_{f}(F)=\sum_{i=1}^{p}\left[F_{i i}\right]$ where $F_{i j}$ are the entries of $F$.

Proof. If $p=1$, then $\operatorname{Tr}_{f}: \Lambda \rightarrow \mathcal{R}(f)$ is just the projection $\lambda \rightarrow[\lambda]$. Let $I_{p}$ be the $p \times p$ square matrix with the diagonal entries equal to $1 \in \Lambda$ and with the non-diagonal equal to 0 . Let 0 be any matrix of zeros.

If $A \in \mathcal{M}_{p, p}, B \in \mathcal{M}_{q, q}, X \in \mathcal{M}_{p, q}$ and $Y \in \mathcal{M}_{q, p}$ then

$\operatorname{Tr}_{f}\left(\begin{array}{cc}A & X \\ Y & B\end{array}\right)=\operatorname{Tr}_{f}\left(\begin{array}{cc}A & 0 \\ 0 & 0\end{array}\right)+\operatorname{Tr}_{f}\left(\begin{array}{cc}0 & X \\ 0 & 0\end{array}\right)+\operatorname{Tr}_{f}\left(\begin{array}{cc}0 & 0 \\ Y & 0\end{array}\right)+\operatorname{Tr}_{f}\left(\begin{array}{cc}0 & 0 \\ 0 & B\end{array}\right)$

by additivity. But

$$
\operatorname{Tr}_{f}\left(\begin{array}{cc}
0 & X \\
0 & 0
\end{array}\right)=\operatorname{Tr}_{f}\left(\begin{array}{c}
X \\
0
\end{array}\right)\left(\begin{array}{ll}
0 & I_{p}
\end{array}\right)=\operatorname{Tr}_{f}\left(\begin{array}{ll}
0 & I_{p}
\end{array}\right)\left(\begin{array}{c}
X^{f} \\
0
\end{array}\right)=0
$$

and similarly $\operatorname{Tr}_{f}\left(\begin{array}{cc}0 & 0 \\ Y & 0\end{array}\right)=0$. Moreover

$$
\begin{aligned}
\operatorname{Tr}_{f}\left(\begin{array}{cc}
A & 0 \\
0 & 0
\end{array}\right) & =\operatorname{Tr}_{f}\left(\begin{array}{c}
I_{p} \\
0
\end{array}\right)\left(\begin{array}{cc}
A & 0
\end{array}\right) \\
& =\operatorname{Tr}_{f}\left(\begin{array}{cc}
A & 0
\end{array}\right)\left(\begin{array}{c}
I_{p}^{f} \\
0
\end{array}\right)=\operatorname{Tr}_{f}\left(A I_{p}^{f}\right)=\operatorname{Tr}_{f}\left(I_{p} A\right)=\operatorname{Tr}_{f}(A)
\end{aligned}
$$

and similarly $\operatorname{Tr}_{f}\left(\begin{array}{ll}0 & 0 \\ 0 & B\end{array}\right)=\operatorname{Tr}_{f}(B)$. Thus $\operatorname{Tr}_{f}\left(\begin{array}{cc}A & X \\ Y & B\end{array}\right)=\operatorname{Tr}_{f}(A)+\operatorname{Tr}_{f}(B)$ and inductively $\operatorname{Tr}_{f}(F)=\sum_{i=1}^{p}\left[F_{i i}\right]$ as required. It is clear that this function satisfies the hypotheses of the proposition.

Now let us suppose that $M$ is a free finitely generated $\Lambda$-module and $F: M \rightarrow M$ is an $f$-endomorphism. For any choice of a free $\Lambda$-basis of $M$ there is a $p \times p$ matrix $\bar{F}$ with entries in $\Lambda$ representing $F$. Let us define $\operatorname{Tr}_{f}(F):=\operatorname{Tr}_{f}(\bar{F})=\sum_{i=1}^{p}\left[\bar{F}_{i i}\right]$. The definition is consistent: let $\left\{e_{1}, e_{2}, \ldots, e_{p}\right\}$ and $\left\{e_{1}^{\prime}, e_{2}^{\prime}, \ldots, e_{p}^{\prime}\right\}$ be two bases. Let $\bar{F}_{i j}$ and $\bar{F}_{i j}^{\prime}$ be the entries of the matrices $\bar{F}$ and $\bar{F}^{\prime}$ representing $F$ in these two bases. This means 
that $F\left(e_{j}\right)=\sum_{i=1}^{p} e_{i} \bar{F}_{i j}$ and $F\left(e_{j}^{\prime}\right)=\sum_{i=1}^{p} e_{i}^{\prime} \bar{F}_{i j}^{\prime}$. Moreover, $e_{i}^{\prime}=\sum_{h=1}^{p} e_{h} A_{h i}$ for a suitable invertible matrix $A$ with entries $A_{i j} \in \Lambda$. Therefore $F\left(e_{j}^{\prime}\right)=\sum_{i, h=1}^{p} e_{h} A_{h i} \bar{F}_{i j}^{\prime}$ and $F\left(e_{j}^{\prime}\right)=\sum_{i=1}^{p} F\left(e_{i}\right) f\left(A_{i j}\right)=\sum_{i, h=1}^{p} e_{h} \bar{F}_{h i} f\left(A_{i j}\right)$ or equivalently the identity $A \bar{F}^{\prime}=$ $\bar{F} A^{f}$ holds true. Let $A^{-1}$ denote the inverse of $A$. Then $\bar{F}^{\prime}=A^{-1} \bar{F} A^{f}$ and hence $\operatorname{Tr}_{f}\left(\bar{F}^{\prime}\right)=\operatorname{Tr}_{f}\left(A^{-1} \bar{F} A^{f}\right)=\operatorname{Tr}_{f}\left(A A^{-1} \bar{F}\right)=\operatorname{Tr}_{f}(\bar{F})$ for the defining property of trace in proposition 2.2 .

Let $\Lambda$ be a ring and let $M$ be a finitely generated projective right $\Lambda$-module. Let $F: M \rightarrow M$ be an $f$-endomorphism. Then there exists a $\Lambda$-module $Q$ such that $M \oplus Q$ is a free finitely generated $\Lambda$-module and an $f$-endomorphism $F+0: M \oplus Q \rightarrow M \oplus Q$ defined by $(F+0)(x+y)=F(x)$ for all $x \in M$ and $y \in Q$. It is an $f$-endomorphism of free finitely generated modules, hence there is a well-defined trace, and we define $\operatorname{Tr}_{f}(F):=\operatorname{Tr}_{f}(F+0)$.

It does not depend on the choice of $Q$ : if $M \oplus Q^{\prime}$ is also free finitely generated, let us consider the free finitely generated $\Lambda$-module $M \oplus Q \oplus M \oplus Q^{\prime}$ with the $f$-endomorphism $F+0_{Q}+0_{M}+0_{Q^{\prime}}: x+y+z+w \rightarrow F(x)$ for all $x, z \in M, y \in Q$ and $w \in Q^{\prime}$. Using the same argument of the proof of proposition 2.2 it is easy to see that $\operatorname{Tr}_{f}\left(F+0_{Q}\right)=$ $\operatorname{Tr}_{f}\left(F+0_{Q}+0_{M}+0_{Q^{\prime}}\right)=\operatorname{Tr}_{f}\left(F+0_{Q^{\prime}}\right)$ thus it is well defined even in the case where $M$ is a finitely generated projective right $\Lambda$-module.

Proposition 2.3 (Commutativity). Let $\Lambda$ be a ring and $M_{1}, M_{2}$ be two finitely generated projective right $\Lambda$-modules. Let $F: M_{1} \rightarrow M_{2}$ and $G: M_{2} \rightarrow M_{1}$ be respectively an $f$-endomorphism and a g-endomorphism, with $f$ and $g$ endomorphisms of $\Lambda$. Then

$$
f_{*} \operatorname{Tr}_{g f}(G F)=\operatorname{Tr}_{f g}(F G)
$$

where $f_{*}: \mathcal{R}(g f) \rightarrow \mathcal{R}(f g)$ is the homomorphism defined in section 2.1.

Proof. Let $Q_{1}$ and $Q_{2}$ be $\Lambda$-modules such that $M_{1} \oplus Q_{1}$ and $M_{2} \oplus Q_{2}$ are free finitely generated. With the same notation as above, it is easily seen that

$$
\begin{aligned}
& \operatorname{Tr}_{f}\left(\left(G+0_{Q_{2}}\right)\left(F+0_{Q_{1}}\right)\right)=\operatorname{Tr}_{f}(G F), \\
& \operatorname{Tr}_{f}\left(\left(F+0_{Q_{1}}\right)\left(G+0_{Q_{2}}\right)\right)=\operatorname{Tr}_{f}(F G),
\end{aligned}
$$

hence substituting $M_{1}$ with $M_{1} \oplus Q_{1}$ and $M_{2}$ with $M_{2} \oplus Q_{2}$ we can suppose $M_{1}$ and $M_{2}$ to be free. Let $e_{1}, \ldots, e_{p}$ be a free basis of $M_{1}$ and $e_{1}^{\prime}, \ldots, e_{q}^{\prime}$ be a free basis of $M_{2}$. Then $F\left(e_{j}\right)=\sum_{i=1}^{q} e_{i}^{\prime} F_{i j}$ and $G\left(e_{j}^{\prime}\right)=\sum_{i=1}^{p} e_{i} G_{i j}$. Hence

$$
G F\left(e_{j}\right)=\sum_{i=1}^{q} G\left(e_{i}^{\prime}\right) g\left(F_{i j}\right)=\sum_{i=1}^{q} \sum_{h=1}^{p} e_{h} G_{h} i g\left(F_{i j}\right)
$$

and similarly

$$
F G\left(e_{j}^{\prime}\right)=\sum_{i=1}^{p} \sum_{h=1}^{q} e_{h}^{\prime} F_{h i} f\left(F_{i j}\right)
$$

Moreover

$$
f_{*}\left(\operatorname{Tr}_{g f}(G F)\right)=\sum_{i=1}^{q} \sum_{h=1}^{p}\left[f\left(G_{h} i\right) f g\left(F_{i h}\right)\right]=\sum_{i=1}^{q} \sum_{h=1}^{p}\left[F_{i h} f\left(G_{h} i\right)\right]=\operatorname{Tr}_{f g}(F G)
$$

hence the assertion. 
2.3. The Lefschetz number of an $f$-endomorphism of a $\Lambda$-complex. Let $\Lambda$ be a ring and let $\mathcal{C}=\left\{C_{n} \rightarrow \ldots \rightarrow C_{0}\right\}$ be a finite projective $\Lambda$-complex, i.e. a finite-dimensional chain complex of finitely generated projective right $\Lambda$-modules. Let $f: \Lambda \rightarrow \Lambda$ be a given ring endomorphism; an $f$-endomorphism $F: \mathcal{C} \rightarrow \mathcal{C}$ of the $\Lambda$-complex $\mathcal{C}$ is any set of $f$-endomorphisms $F_{n}: C_{i} \rightarrow C_{i}$ for $i=0, \ldots, n$ which commute with the boundary homomorphisms. The traces $\operatorname{Tr}_{f}\left(F_{i}\right)$ are well-defined. The Lefschetz number of $F$ is defined to be

and it is an element of $\mathcal{R}(f)$.

$$
\mathcal{L}(F)=\sum_{q \geq 0}(-1)^{q} \operatorname{Tr}_{f}\left(F_{q}\right)
$$

Proposition 2.4 (Homotopy). If $F, G: C \rightarrow C$ are chain-homotopic $f$-endomorphisms then $\mathcal{L}(F)=\mathcal{L}(G)$.

Proof. Let $d_{i}: C_{i} \rightarrow C_{i+1}$ and $\partial_{i}: C_{i+1} \rightarrow C_{i}$ for $i=1, \ldots, n$ be the chain homotopy between $F$ and $G$ and the boundary homomorphisms; let us recall that $d_{i}$ simply is a $\Lambda$-homomorphism of $\Lambda$-modules. By additivity $\mathcal{L}(F)-\mathcal{L}(G)=\mathcal{L}(F-G)$ and

$$
\begin{aligned}
\mathcal{L}(F-G) & =\sum_{q \geq 0}(-1)^{q}\left(\operatorname{Tr}_{f}\left(\partial_{i+1} d_{i}\right)+\operatorname{Tr}_{f}\left(d_{i-1} \partial_{i}\right)\right) \\
& =\sum_{q \geq 0}(-1)^{q}\left(\operatorname{Tr}_{f}\left(\partial_{i+1} d_{i}\right)-\operatorname{Tr}_{f}\left(d_{i} \partial_{i+1}\right)\right)=0
\end{aligned}
$$

by commutativity of $\operatorname{Tr}_{f}$ as in proposition 2.3. Hence $\mathcal{L}(F)=\mathcal{L}(g)$.

Proposition 2.5 (Commutativity). Let $\Lambda$ be a ring and $C, C^{\prime}$ be two chain complexes of finitely generated projective right $\Lambda$-modules. Let $F: C \rightarrow C^{\prime}$ and $G: C^{\prime} \rightarrow C$ be respectively an $f$-endomorphism and a $g$-endomorphism, with $f$ and $g$ endomorphisms of $\Lambda$. Then

$$
f_{*} \mathcal{L}(G F)=\mathcal{L}(F G)
$$

where $f_{*}: \mathcal{R}(g f) \rightarrow \mathcal{R}(f g)$ is the homomorphism defined in section 2.1.

Proof. It is a trivial corollary of 2.3 .

\section{Topological preliminaries}

3.1. The generalized Lefschetz number of a continuous self-map on a finite $C W$ complex. Let $X$ be a finite $C W$-complex. We are not requiring it to be connected. Let $X^{1}, X^{2}, \ldots, X^{p}$ be its connected components and let $x^{i} \in X^{i}$ be a base point of $X^{i}$ for each $i=1, \ldots, p$. Let $\Lambda(X)$ denote the free abelian group generated by the elements of the fundamental groups of these components, i.e.

$$
\Lambda(X):=\mathbf{Z} \pi_{1}\left(X^{1}, x^{1}\right) \oplus \mathbf{Z} \pi_{1}\left(X^{2}, x^{2}\right) \oplus \ldots \oplus \mathbf{Z} \pi_{1}\left(X^{p}, x^{p}\right)
$$

and let the product in $\Lambda(X)$ be defined by the linear extension of

$$
g h= \begin{cases}g h & \text { if } g, h \in \pi_{1}\left(X^{i}, x^{i}\right) \text { for some } i, \\ 0 & \text { if } g \in \pi_{1}\left(X^{i}, x^{i}\right) \text { and } h \in \pi_{1}\left(X^{j}, x^{j}\right) \text { with } i \neq j .\end{cases}
$$

If $X$ is connected then $\Lambda(X)=\mathbf{Z} \pi_{1}(X)$ is simply the group ring of the fundamental group of $X$. 
Let $1_{i}$ denote the constant loop in $\pi_{1}\left(X^{i}, x^{i}\right)$. Then $1:=\sum_{i=1}^{p} 1_{i}$ is the unit element of $\Lambda$.

Let $f: X \rightarrow X$ be a self-map. Let $J:=\mathbf{Z}_{p} \times I$ be the cartesian product of the set of the first $p$ integers $\mathbf{Z}_{p}=\{1,2, \ldots, p\}$ with discrete topology and the unit interval $I=[0,1]$. A continuous map $w: J \rightarrow X$ is called a base multipath if for all $j=1, \ldots, p$ there exist $j^{\prime}$ such that

$$
w(j, 0)=x_{j^{\prime}}, \quad w(j, 1)=f\left(x_{j}\right) .
$$

Let us note that $j^{\prime}$ is uniquely determined once we have the second identity; it is because $X^{j^{\prime}}$ is connected. We say that the self-map $f$ is multipath-based if a base multipath $w$ has been chosen and we denote it by $(f, w)$. Up to rearranging indices it is always possible to assume that $f\left(x^{i}\right) \in X^{i}$ for $i=1, \ldots, p_{0}$ and $f\left(x^{i}\right) \in X^{i^{\prime}}$ with $i^{\prime} \neq i$ for $i=p_{0}+1, \ldots, p$.

For any multipath-based self-map $(f, w): X \rightarrow X$ there is an induced endomorphism $f_{\Lambda}: \Lambda(X) \rightarrow \Lambda(X)$ defined as the linear extension of

$$
f_{\Lambda}\left(g_{i}\right)=1_{i} f_{\pi}\left(g_{i}\right)
$$

if $g_{i} \in \pi_{1}\left(X^{i}, x^{i}\right)$ and $\left.f_{\pi}: \pi_{1}\left(X^{i}, x^{i}\right) \rightarrow \pi_{(} X^{i^{\prime}}, x^{i^{\prime}}\right)$ is defined by

$$
f_{\pi}(\alpha)=w(i,-) f(\alpha) w(i,-)^{-1}
$$

where $\alpha:(I, \partial I) \rightarrow\left(X^{i}, x^{i}\right)$ is a loop in $X^{i}$ and $w(i,-): I \rightarrow\{i\} \times I \stackrel{w}{\rightarrow} X^{i^{\prime}}$ is the path in $X^{i^{\prime}}$ from $x^{i^{\prime}}$ to $f\left(x^{i}\right)$ we have previously chosen. In other words $f_{\Lambda}\left(g_{i}\right)=f_{\pi}\left(g_{i}\right)$ if $1 \leq i \leq p_{0}$ and $f_{\Lambda}\left(g_{i}\right)=0$ if $p_{0}+1 \leq i \leq p$.

Let $\tilde{X}$ be the universal covering space of $X$. It is the disjoint union of the universal covering spaces of $X^{1}, \ldots, X^{p}$. If the set of paths $P X:=\left\{\lambda:(I,\{0\}) \rightarrow\left(X,\left\{x^{1}, \ldots, x^{p}\right\}\right)\right\}$ is endowed with the compact-open topology, then $\tilde{X}$ is the quotient space of $P X$ under the relation of homotopy equivalence relative to endpoints. Therefore we can view a point in $\tilde{X}$ as a homotopy class of paths $[\lambda]$. For any $g \in \pi_{1}\left(X_{i}, x_{i}\right)$ let

$$
[\lambda] g= \begin{cases}{\left[g^{-1} \lambda\right]} & \text { if } \lambda(0)=x_{i} \\ {[\lambda]} & \text { if } \lambda(0) \neq x_{i}\end{cases}
$$

be defined as above. The map $[\lambda] \rightarrow[\lambda] g$ is the cellular homeomorphism of $\tilde{X}$ induced by $g$.

For every integer $q \geq 0$ let $C_{q}(\tilde{X})$ denote the $q$-th cellular chain group $C_{q}(\tilde{X})=$ $H_{q}\left(\tilde{X}^{(q)}, \tilde{X}^{(q-1)} ; \mathbf{Z}\right)$ where $\tilde{X}^{(q)}$ is the $q$-dimensional skeleton of $\tilde{X}$ for all positive integers $q$. We know that $C_{q}(\tilde{X})=C_{q}\left(\tilde{X}^{1}\right) \oplus \ldots \oplus C_{q}\left(\tilde{X}^{p}\right)$.

Let $\Lambda(X)$ act on $C_{q}(\tilde{X})$ on the right by extending linearly the function defined for each $x \in C_{q}\left(\tilde{X}^{i}\right)$ and $g \in \pi_{1}\left(X^{j}, x^{j}\right)$ by

$$
x g= \begin{cases}C_{q}(g)(x) & \text { if } i=j, \\ 0 & \text { if } i \neq j,\end{cases}
$$

where $C_{q}(g): C_{q}\left(\tilde{X}^{i}\right) \rightarrow C_{q}\left(\tilde{X}^{i}\right)$ is the homomorphism induced by the map $[\lambda] \in \tilde{X}^{1} \rightarrow$ $\left[g^{-1} \lambda\right] \in \tilde{X}^{1}$. Thus $C_{q}(\tilde{X})$ is a right $\Lambda$-module. If $X$ is connected, it is free and finitely generated. In our general setting a weaker proposition holds.

Proposition 3.1. The $q$-th cellular chain group $C_{q}(\tilde{X})$ is a finitely generated projective right $\Lambda(X)$-module. 
Proof. We have to prove that each $C_{q}\left(\tilde{X}^{i}\right)$ is a finitely generated projective $\Lambda(X)$ module. We already know that for each $i=1, \ldots, p, C_{q}\left(\tilde{X}^{i}\right)$ is a free finitely generated $\Lambda\left(X^{i}\right)$-module. Let $\left\{e_{1}, \ldots, e_{k}\right\}$ be a free basis. Just by taking the projection $p r_{i}: \Lambda(\tilde{X})=$ $\Lambda\left(\tilde{X}^{1}\right) \oplus \ldots \oplus \Lambda\left(\tilde{X}^{p}\right) \rightarrow \Lambda\left(\tilde{X}^{i}\right)$ we can define a right action of $\Lambda(X)$ on $C_{q}\left(\tilde{X}^{i}\right)$ by $x \lambda:=x \operatorname{pr}_{i}(\lambda)$ for each $x \in C_{q}\left(\tilde{X}^{i}\right)$ and each $\lambda \in \Lambda(X)$. Hence $C_{q}\left(\tilde{X}^{i}\right)$ is a right finitely generated $\Lambda(X)$-module. Let $\Lambda^{i}:=\sum_{j \neq i} \Lambda\left(\tilde{X}^{j}\right)$ be the complement of $\Lambda\left(\tilde{X}^{i}\right)$ in $\Lambda(X)$. Let $Q_{i}$ be the direct sum of $k$ copies of $\Lambda^{i}$. Let $\Lambda(X)$ act on $Q_{i}$ by the usual ring product in $\Lambda(X)$ and distributive law. Therefore

$$
C_{q}\left(\tilde{X}^{i}\right) \oplus Q_{i} \cong \bigoplus_{u=1}^{k}\left(\Lambda\left(\tilde{X}^{u}\right) \oplus \Lambda^{u}\right)
$$

and hence it is a free finitely generated right $\Lambda(X)$-module.

If $(f, w)$ is a multipath-based cellular self-map of $X$ then there is a canonical cellular lifting of $(f, w)$, namely $\tilde{f}: \tilde{X} \rightarrow \tilde{X}$, defined by $\tilde{f}([\lambda])=[w(i,-) f(\lambda)]$ for each path $\lambda:(I, 0) \rightarrow\left(X^{i}, x^{i}\right)$. It induces an endomorphism $C_{q}(\tilde{f}): C_{q}(\tilde{X}) \rightarrow C_{q}(\tilde{X})$ at the cellular chain group level. Let $P: C_{q}(\tilde{X})=\bigoplus_{i=1}^{p} C_{q}\left(\tilde{X}^{i}\right) \rightarrow C_{q}(\tilde{X})$ be the homomorphism defined by

$$
P(x):= \begin{cases}x & \text { if } x \in C_{q}\left(\tilde{X}^{i}\right) \text { with } i \leq p_{0} \\ 0 & \text { if } x \in C_{q}\left(\tilde{X}^{i}\right) \text { with } i \geq p_{0}+1 .\end{cases}
$$

It will be called the projection homomorphism for $C_{q}(\tilde{X})$.

It is easy to see that the composition $C_{q}(\tilde{f}) P$ is an $f_{\Lambda}$-endomorphism, where $f_{\Lambda}$ : $\Lambda(X) \rightarrow \Lambda(X)$ is defined as above. Therefore we can define the generalized Lefschetz number of the multipath-based cellular self-map $(f, w)$ as the Lefschetz number of $C_{q}(\tilde{f}) P$

$$
\mathcal{L}(f, w)=\sum_{q \geq 0}(-1)^{q} \operatorname{Tr}_{f_{\Lambda}}\left[C_{q}(\tilde{f}) P\right]
$$

which is an element of $\mathcal{R}\left(f_{\Lambda}\right)$ (see $\left.[\mathrm{H}],[\mathrm{FH}]\right)$. Let us note that when $p=1$ this is the generalized Lefschetz number as defined in $[\mathrm{H}]$.

It is expected that $\mathcal{L}(f, w)$ is independent of the base multipath $w$ and depends only on the homotopy class of $f$. This is truly the case: for $i=1, \ldots, p$, let $x^{i} \in X^{i}$ be another base point and $w^{\prime}: J \rightarrow X$ another corresponding base multipath. The paths $w(i,-)$ and $w^{\prime}(i,-)$ will be denoted simply with $w_{i}$ and $w_{i}^{\prime}$. Let $\tilde{X}^{\prime}$ denote the universal covering space pointed at $x^{\prime 1}, \ldots, x^{\prime p}$ and $\tilde{f}^{\prime}: \tilde{X}^{\prime} \rightarrow \tilde{X}^{\prime}$ the canonical lifting of $f$ at $\tilde{X}^{\prime}$. The rings

$$
\begin{aligned}
\Lambda(X) & :=\mathbf{Z} \pi_{1}\left(X^{1}, x^{1}\right) \oplus \mathbf{Z} \pi_{1}\left(X^{2}, x^{2}\right) \oplus \ldots \oplus \mathbf{Z} \pi_{1}\left(X^{p}, x^{p}\right), \\
\Lambda^{\prime}(X) & :=\mathbf{Z} \pi_{1}\left(X^{1}, x^{\prime 1}\right) \oplus \mathbf{Z} \pi_{1}\left(X^{2}, x^{\prime 2}\right) \oplus \ldots \oplus \mathbf{Z} \pi_{1}\left(X^{p}, x^{\prime p}\right)
\end{aligned}
$$

are given. For each $i=1, \ldots, p$, let $\gamma_{i}:(I, 0,1) \rightarrow\left(X^{i}, x^{i}, x^{i}\right)$ be a continuous path from $x^{i}$ to $x^{\prime i}$. Let $\varphi_{1}, \varphi_{2}: \Lambda(X) \rightarrow \Lambda^{\prime}(X)$ be defined by extending linearly $\varphi_{1}(g):=\gamma_{i}^{-1} g \gamma_{i}$ and $\varphi_{2}(g):=w_{i}^{\prime} f\left(\gamma_{i}^{-1}\right) w_{i}^{-1} g w_{i} f\left(\gamma_{i}\right) w_{i}^{-1}$ if $g \in \pi_{1}\left(X^{i}, x^{i}\right) \subseteq \Lambda(X)$ and $f\left(x^{i}\right) \in X^{i}$. Otherwise $\varphi_{1}(g):=\varphi_{2}(g):=\gamma_{i}^{-1} g \gamma_{i}$. It is easy to see that $\varphi_{2} f_{\Lambda}=f_{\Lambda^{\prime}} \varphi_{1}$.

Let us note that $\Lambda(X)$ and $\Lambda^{\prime}(X)$ are well-decomposed (see section 2.2) into

$$
\bigoplus_{i=1}^{p} \Lambda\left(X^{i}\right) \text { and } \bigoplus_{i=1}^{p} \Lambda^{\prime}\left(X^{i}\right)
$$


respectively, and that if we set

$$
\theta_{i}:=\gamma_{i}^{-1} w_{i} f\left(\gamma_{i}\right) w_{i}^{\prime-1}
$$

if $f\left(x_{i}\right) \in X_{i}$ and otherwise $\theta_{i}:=1$, then the identity $\theta_{i} \varphi_{2}\left(\lambda_{i}\right)=\varphi_{1}\left(\lambda_{i}\right) \theta_{i}$ holds true for all $\lambda_{i} \in \Lambda\left(X_{i}\right)$. Therefore there exists a well-defined group homomorphism $\theta_{*}: \mathcal{R}\left(f_{\Lambda}\right) \rightarrow$ $\mathcal{R}\left(f_{\Lambda^{\prime}}\right)$ defined as in section 2.1 .

Let $\Phi_{1}, \Phi_{2}: \tilde{X} \rightarrow \tilde{X}^{\prime}$ be the homeomorphisms defined by $\Phi_{1}([\lambda]):=\left[\gamma_{i}^{-1} \lambda\right]$ if $\lambda(0)=$ $x^{i}$ and $\Phi_{2}([\lambda]):=\left[w_{i}^{\prime} f\left(\gamma_{i}^{-1}\right) w_{i}^{-1} \lambda\right]$ if $\lambda(0)=x^{i}$ and $f\left(x^{i}\right) \in X^{i}$; otherwise $\Phi_{2}([\lambda]):=$ $\Phi_{1}([\lambda])$. We can see that at the cellular complex level $C_{q}\left(\Phi_{2}\right) C_{q}(\tilde{f}) P=C_{q}\left(\tilde{f}^{\prime}\right) P^{\prime} C_{q}\left(\Phi_{1}\right)$ where $P: C_{q}(\tilde{X}) \rightarrow C_{q}(\tilde{X})$ and $P^{\prime}: C_{q}\left(\tilde{X}^{\prime}\right) \rightarrow C_{q}\left(\tilde{X}^{\prime}\right)$ are defined as above. Moreover $C_{q}\left(\Phi_{1}\right)$ and $C_{q}\left(\Phi_{2}\right)$ are a $\varphi_{1}$ and $\varphi_{2}$-homomorphism respectively which satisfy the identity

$$
C_{q}\left(\Phi_{2}\right)(x)=C_{q}\left(\Phi_{1}\right)(x) \cdot \theta_{i}
$$

for all $x \in C_{q}\left(\tilde{X}_{i}\right)$. We have

$$
C_{q}\left(\tilde{f}^{\prime}\right) P^{\prime}=C_{q}\left(\phi_{2}\right) C_{q}(\tilde{f}) P C_{q}\left(\Phi_{1}^{-1}\right)
$$

and hence by commutativity

$$
\mathcal{L}\left(f, w^{\prime}\right)=\mathcal{L}\left(C_{q}\left(\tilde{f}^{\prime}\right) P^{\prime}\right)=\varphi_{1 *} \mathcal{L}\left(C_{q}\left(\Phi_{1}^{-1}\right) C_{q}\left(\Phi_{2}\right) C_{q}(\tilde{f}) P\right) .
$$

But $C_{q}\left(\Phi_{2}\right)(x)=C_{q}\left(\phi_{1}\right)(x) \cdot \theta_{i}$ for each $x \in C_{q}\left(\tilde{X}_{i}\right)$; therefore

$$
C_{q}\left(\Phi_{1}^{-1}\right) C_{q}\left(\phi_{2}\right)(x)=x \cdot \varphi_{1}^{-1}\left(\theta_{i}\right)
$$

for all $x \in C_{q}\left(\tilde{X}_{i}\right)$. Hence

$$
\varphi_{1 *} \mathcal{L}\left(C_{q}\left(\Phi_{1}^{-1}\right) C_{q}\left(\Phi_{2}\right) C_{q}(\tilde{f}) P\right)=\theta_{*} \mathcal{L}\left(C_{q}(\tilde{f}) P\right)=\theta_{*} \mathcal{L}(f, w)
$$

where $\theta_{*}$ is the isomorphism defined in section 2.1.

If $H: f \sim f^{\prime}$ is a cellular homotopy then it can be shown that $H$ induces an isomorphism $H_{*}: \mathcal{R}\left(f_{\Lambda}\right) \rightarrow \mathcal{R}\left(f_{\Lambda}^{\prime}\right)$ such that $\mathcal{L}\left(f^{\prime}, w^{\prime}\right)=H_{*} \mathcal{L}(f, w)$ for suitable base multipaths $w$ and $w^{\prime} . H_{*}$ can be defined by considering the chain homotopy at the chain complex level, in the same way as in $[\mathrm{H}]$. We prefer to give a slightly different proof which follows the lines of $[\mathrm{F}]$. Let $\bar{H}: X \times I \rightarrow X \times I$ be a cellular approximation of the fat homotopy (cf. $[\mathrm{J}],[\mathrm{B}])$ such that $\bar{H}(-, 0)=f$ and $\bar{H}(-, 1)=f^{\prime}$. Let $w, w^{\prime}$ be base multipaths for $f$ and $f^{\prime}$ respectively with the same base points $x^{1}, \ldots, x^{p}$. It is easy to see that, if $i_{0}, i_{1}: X \rightarrow X \times I$ are defined by $i_{0}(x):=(x, 0)$ and $i_{1}(x):=(x, 1)$ for all $x \in X$, then $i_{0 *}(\mathcal{L}(f, w))=\mathcal{L}\left(\bar{H}, i_{0}(w)\right)$ and $i_{1 *}\left(\mathcal{L}\left(f^{\prime}, w^{\prime}\right)\right)=\mathcal{L}\left(\bar{H}, i_{1}\left(w^{\prime}\right)\right)$. Let $\gamma_{i}:(I, 0,1) \rightarrow$ $\left(X \times I,\left(x^{i}, 0\right),\left(x^{i}, 1\right)\right)$ be the vertical path from $\left(x^{i}, 0\right)$ to $\left(x^{i}, 1\right)$. Then for the previous arguments there exists an isomorphism $\theta_{*}$ such that $\theta_{*}\left(\mathcal{L}\left(\bar{H}, i_{0}(w)\right)\right)=\mathcal{L}\left(\bar{H}, i_{1}\left(w^{\prime}\right)\right)$. We can therefore define

$$
H_{*}:=i_{1 *}^{-1} \theta_{*} i_{0 *}: \mathcal{R}\left(f_{\Lambda}\right) \rightarrow \mathcal{R}\left(f_{\Lambda}^{\prime}\right)
$$

which coincides with the one defined in $[\mathrm{F}]$ if $X$ is connected.

Let us remark that such an isomorphism exists even if $f$ is not cellular; in this case $\mathcal{L}(f, w)$ is not yet defined, but at the $\mathcal{R}\left(f_{\Lambda}\right)$-level everything works. So if $(f, w)$ is not cellular we can define $\mathcal{L}(f, w):=H_{*} \mathcal{L}\left(f^{\prime}, w^{\prime}\right)$ where $f^{\prime}: X \rightarrow X$ is any cellular approximation of $f$ and $H$ is the homotopy between $f$ and $f^{\prime}$ and $w^{\prime}$ is a base multipath for $f^{\prime}$; it turns out that $\mathcal{L}(f, w)$ does not depend on the choice of $f^{\prime}$. 
Proposition 3.2. Let $X^{1}, \ldots, X^{p}$ be the connected components of $X$, with base points $x^{i} \in X^{i}$. Let us suppose that $f\left(x^{i}\right) \in X^{i}$ for $i=1, \ldots, p_{0}$ and $f\left(x^{i}\right) \in X^{j}$ with $j \neq i$ for $i=p_{0}, \ldots, p$. Let $w: J \rightarrow X$ be a base multipath for $f$, and $w_{i}:=w(i,-)$. Let $f_{i}: X^{i} \rightarrow X^{i}$ be the restriction of $f$ to $X^{i}$ for $i=1, \ldots, p_{0}$. Then

$$
\mathcal{R}\left(f_{\Lambda}\right)=\bigoplus_{i=1}^{p_{0}} \mathcal{R}\left(f_{i_{\Lambda}}\left(X^{i}\right)\right) \quad \text { and } \quad \mathcal{L}(f, w)=\sum_{i=1}^{p_{0}} \mathcal{L}\left(f_{i}, w_{i}\right)
$$

where $\mathcal{L}\left(f_{i}, w_{i}\right)$ is the generalized Lefschetz number of $f_{i}: X^{i} \rightarrow X^{i}$.

Proof. It is trivial to check that $\Lambda(X)$ is well-decomposed into $\Lambda\left(X_{1}\right), \ldots, \Lambda\left(X_{p}\right)$. Therefore

$$
\mathcal{R}\left(f_{\Lambda}\right)=\bigoplus_{i=1}^{p} \mathcal{R}\left(\left.f\right|_{\Lambda\left(X_{i}\right)}\right)
$$

but for $i=1 \cdot p_{0}, \mathcal{R}\left(\left.f\right|_{\Lambda\left(X_{i}\right)}\right)=\mathcal{R}\left(f_{i_{\Lambda}\left(X_{i}\right)}\right)$ and for $i=p_{0}, \ldots, p, \mathcal{R}\left(\left.f\right|_{\Lambda\left(X_{i}\right)}\right)=0$. Hence the first identity.

Now let $e_{1}, \ldots, e_{k}$ be a free $\Lambda\left(X^{1}\right)$-basis for $C_{q}\left(\tilde{X}^{1}\right)$ which is a finitely generated free right $\Lambda\left(X^{1}\right)$-module, because $X^{1}$ is connected; let us remark that $\Lambda\left(X^{1}\right)=\mathbf{Z} \pi_{1}\left(X^{1}, x^{1}\right)$. Then $C_{q}\left(\tilde{f}_{1}\right)\left(e_{j}\right)=\sum_{h=1}^{k} e_{h} F_{h j}^{1}$ for suitable $F_{h j}^{1} \in \Lambda\left(X^{1}\right)$. Now we can take a $\Lambda(X)$ module $Q_{1}$ such that $C_{q}\left(\tilde{X}^{1}\right) \oplus Q_{1}$ is a free $\Lambda(X)$-module with $e_{1}, \ldots, e_{k}$ as a free $\Lambda(X)$ basis, as done in proposition 3.1. This argument can be applied to each $j=1, \ldots, p_{0}$. Therefore, if $i_{j}: \mathcal{R}\left(f_{j_{\Lambda}\left(X_{j}\right)}\right) \rightarrow \mathcal{R}\left(f_{\Lambda(X)}\right)$ is the obvious inclusion, we have that

$$
\operatorname{Tr}\left(C_{q}(\tilde{f}) P\right)=\sum_{j=1}^{p_{0}} i_{j} \operatorname{Tr}\left(C_{q}\left(\tilde{f}_{j}\right)\right)
$$

and taking alternating sums,

$$
\mathcal{L}\left(C_{q}(\tilde{f}) P\right)=\sum_{j=1}^{p_{0}} \mathcal{L}\left(C_{q}\left(\tilde{f}_{j}\right)\right)
$$

and so the conclusion follows.

For each $i=1, \ldots, p_{0}$ there exists a coordinate function $\operatorname{cd}_{i}: \operatorname{Fix}\left(f_{i}\right) \rightarrow \mathcal{R}\left(f_{i_{\Lambda}\left(X^{i}\right)}\right)$ defined by $\operatorname{cd}_{i}(y):=\lambda f_{i}\left(\lambda^{-1}\right) w_{i}^{-1}$ for all $y \in \operatorname{Fix}\left(f_{i}\right)$ with a path $\lambda:(I, 0,1) \rightarrow\left(X^{i}, x^{i}, y\right)$ (see e.g. $[\mathrm{B}],[\mathrm{J}]$ ). The main theorem of $[\mathrm{H}]$ states that

$$
\mathcal{L}\left(f_{i}, w_{i}\right)=\sum_{x \in \operatorname{Fix}\left(f_{i}\right)} \operatorname{Ind}\left(f_{i}, x\right) \dot{\operatorname{cd}}_{i}(x)
$$

where $\operatorname{Ind}\left(f_{i}, x\right)$ is the index of the fixed point $x$, and $\operatorname{Fix}\left(f_{i}\right):=\left\{y \in X_{i} \mid f_{i}(y)=y\right\}$ is the fixed point set for $f_{i}$. We can always assume $\operatorname{Fix}(f)$ to be a finite subset of $X$. The same formula holds for $f: X \rightarrow X$; if $\operatorname{cd}: \operatorname{Fix}(f) \rightarrow \mathcal{R}(f)$ is defined by $\operatorname{cd}(y):=\operatorname{cd}_{i}(y)$ for every $y \in X_{i} \cap \operatorname{Fix}(f)$ then we have the identity

$$
\mathcal{L}(f, w)=\sum_{x \in \operatorname{Fix}(f)} \operatorname{Ind}(f, x) \cdot \operatorname{cd}(x) .
$$

Let us recall that the number of nontrivial distinct free generators of $\mathcal{R}\left(f_{i}, w_{i}\right)$ which have to be used in writing $\mathcal{L}\left(f_{i}, w_{i}\right)$ is the Nielsen number $N\left(f_{i}\right)$ of the map $f_{i}$ as defined in 
$[\mathrm{B}],[\mathrm{J}]$. The same is true for $f: X \rightarrow X$ in the sense that we can define the Nielsen number of $f, N(f)$, to be the number of nontrivial distinct free generators of $\mathcal{R}(f)$ which have to be used in writing $\mathcal{L}(f, w)$. It is the sum $N(f)=\sum_{i=1}^{p_{0}} N\left(f_{i}\right)$ of the Nielsen numbers of the restrictions $f_{i}: X_{i} \rightarrow X_{i}$. In the same way the inequality $N(f) \leq \# \operatorname{Fix}(f)$ holds. The Nielsen number naturally continues to be a lower bound of the number of fixed points of the self-map $f$.

3.2. Pushout maps. Let $A, X_{1}$ and $X_{2}$ be finite, not necessarily connected $C W$ complexes. Let $i_{1}: A \rightarrow X_{1}$ and $i_{2}: A \rightarrow X_{2}$ be cellular continuous maps. Then the pushout space $X:=X_{1} \sqcup_{A} X_{2}$ of $X_{1}$ and $X_{2}$ via $i_{1}$ and $i_{2}$, or the pushout space of $i_{1}$ and $i_{2}$ for short, is the set of all equivalence classes of the topological sum $X_{1} \sqcup X_{2}$ under the equivalence relation generated by $x_{1} \sim x_{2} \Longleftrightarrow(\exists a \in A) x_{1}=i_{1}(a), x_{2}=i_{2}(a)$. It can be shown that $X$ is a finite $C W$-complex. Let $q: X_{1} \sqcup X_{2} \rightarrow X_{1} \sqcup_{A} X_{2}$ be the identification function and define $j_{1}: X_{1} \rightarrow X$ and $j_{2}: X_{2} \rightarrow X$ as the compositions of $q$ with the inclusions of $X_{1}$ and $X_{2}$ in $X_{1} \sqcup X_{2}$. For more details see [P]. The main property of a pushout space is the universal property: given two maps with the same codomain $h_{1}: X_{1} \rightarrow Z, h_{2}: X_{2} \rightarrow Z$ such that $h_{1} i_{1}=h_{2} i_{2}$, there exists a unique $l: X \rightarrow Z$ such that $l j_{1}=h_{1}$ and $l j_{2}=h_{2}$.

Here is a list of very common pushout-type constructions.

EXAmple 3. Union spaces. If $X=X_{1} \cup X_{2}$ is the union of two subcomplexes $X_{1}$ and $X_{2}$, then $X=X_{1} \sqcup_{A} X_{2}$ where $A=X_{1} \cap X_{2}$ and $i_{1}: A \rightarrow X_{1}, i_{2}: A \rightarrow X_{2}$ are the inclusions. For any cellular self-maps $f_{1}$ and $f_{2}$ of $X_{1}$ and $X_{2}$ that coincide on the common intersection $A$, there exists the extended map $f: X \rightarrow X$ which is the pushout map of $f_{1}$ and $f_{2}$ via $f_{A}$.

Example 4. Quotient spaces. Let $(X, A)$ be a pair of finite $C W$-complexes. Then the quotient space $X / A$ is the pushout space of $i_{1}: A \rightarrow X$ and $i_{2}: A \rightarrow\{*\}$ where $i_{1}$ is the inclusion and $i_{2}$ the constant map.

Example 5. One-point unions. The one-point union of two spaces $X_{1}$ and $X_{2}$ is simply the pushout space of $i_{1}:\{*\} \rightarrow X_{1}$ and $i_{2}:\{*\} \rightarrow X_{2}$.

Example 6. Connected sums. Let $M_{1}$ and $M_{2}$ be two compact triangulated $n$-manifolds. Let $X_{1}:=M_{1}-D^{n}$ and $X_{2}:=M_{2}-D^{n}$ be the manifolds minus an open ball $D^{n}$, and $A:=\partial \bar{D}^{n}$. Then the connected sum $M_{1} \# M_{2}$ is the pushout space of $i_{1}: A \rightarrow X_{1}$ and $i_{2}: A \rightarrow X_{2}$ if $i_{1}$ and $i_{2}$ are the natural inclusions of $\partial \bar{D}^{n}$ in $M_{1}$ and $M_{2}$.

EXAMPLE 7. Mapping cylinder. Let $i_{2}: A \rightarrow X_{2}$ be any cellular map. The pushout space of $i_{0}: A \rightarrow A \times I$ and $i_{2}$, where $i_{0}(a):=(a, 0)(\forall a \in A)$, is called the mapping cylinder $M\left(i_{2}\right)$ of $i_{2}$ and is useful in the proof of the pushout formula of this paper.

ExAmple 8. Mapping torus. Let $Y$ be a finite $C W$-complex and $f: Y \rightarrow Y$ be a self-map. Let $A:=Y \times \partial I, X_{1}:=Y \times I$ and $X_{2}:=Y$. If $i_{1}$ is the inclusion $A \rightarrow X_{1}$ and $i_{2}$ is defined by $i_{2}(y, 0)=y$ and $i_{2}(y, 1)=f(y)$ for all $y \in Y$, then the pushout space is the mapping torus $T_{f}$ of $f$, as defined in [J], [J1].

Now let us consider cellular self-maps $f_{A}: A \rightarrow A, f_{1}: X_{1} \rightarrow X_{1}$ and $f_{2}: X_{2} \rightarrow X_{2}$ such that $i_{1} f_{A}=f_{1} i_{1}$ and $i_{2} f_{A}=f_{2} i_{2}$. There exists a unique cellular self-map $f: X \rightarrow X$ 
defined on the pushout space $X$ such that the following diagram is commutative:

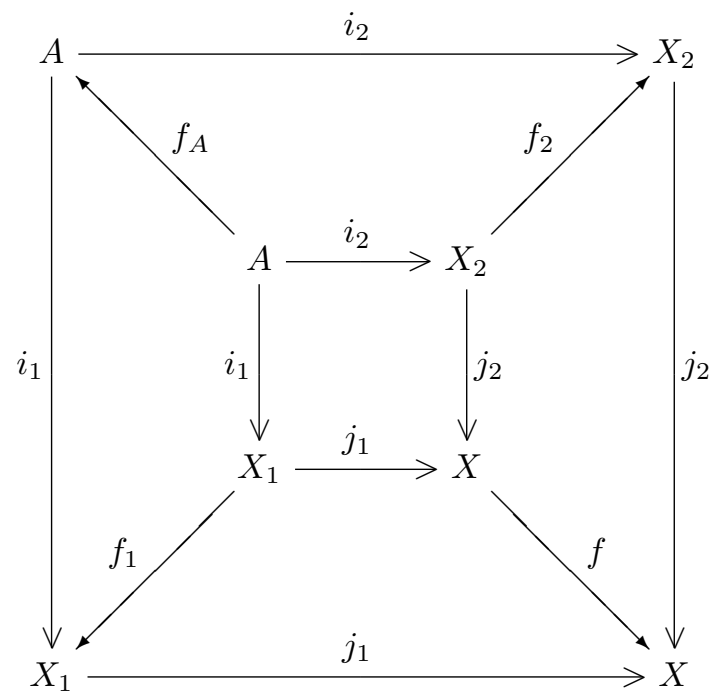

The map $f$ is called the pushout map of $f_{1}$ and $f_{2}$ via $f_{A}$ and can be denoted by $f_{1} \sqcup_{f_{A}} f_{2}$ in analogy with topological spaces.

Let $w_{A}, w_{1}, w_{2}$ and $w$ be base multipaths for $f_{A}, f_{1}, f_{2}$ and $f$. We wish to show that there exist well-defined homomorphisms $i_{* 1}: \mathcal{R}\left(f_{A}, w_{A}\right) \rightarrow \mathcal{R}\left(f_{1}, w_{1}\right), i_{2 *}: \mathcal{R}\left(f_{A}, w_{A}\right) \rightarrow$ $\mathcal{R}\left(f_{2}, w_{2}\right), j_{1 *}: \mathcal{R}\left(f_{1}, w_{1}\right) \rightarrow \mathcal{R}(f, w)$ and $j_{2 *}: \mathcal{R}\left(f_{2}, w_{2}\right) \rightarrow \mathcal{R}(f, w)$ such that $j_{2 *} i_{2 *}=$ $j_{1 *} i_{1 *}$. Let us consider one of the squares of the previous diagram, e.g.

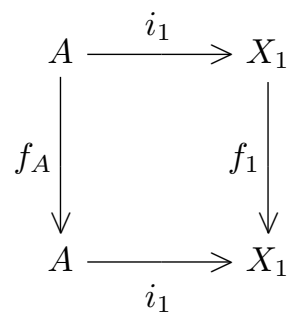

where $A^{1}, \ldots, A^{p}$ are the connected components of $A$ and $X_{1}^{1}, \ldots, X_{1}^{q}$ those of $X_{1}$. Let $a^{1} \in A^{1}, \ldots a^{p} \in A^{p}, x_{1}^{1} \in X_{1}^{1}, \ldots, x_{1}^{q} \in X_{1}^{q}$ be the base points. For each $i=1, \ldots, p$, let us choose a path $\gamma_{i}:(I, 0,1) \rightarrow\left(X_{1}, i_{1}\left(a^{i}\right), x_{1}^{i}\right)$. The diagram

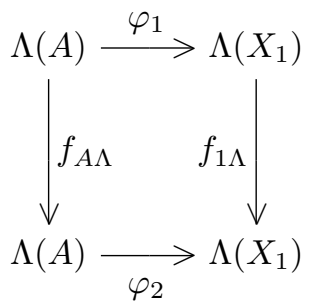

commutes, if $\varphi_{1}$ and $\varphi_{2}$ are defined by extending linearly

$$
\varphi_{1}(g):=\gamma_{i}^{-1} i_{1}(g) \gamma_{i}, \quad \varphi_{2}(g):=w_{1} f_{1}\left(\gamma_{i}^{-1}\right) i_{1}\left(w_{A}^{-1} g w_{A}\right) f_{1}\left(\gamma_{i}\right) w_{1}^{-1}
$$


if $g \in \pi_{1}\left(A^{i}, a^{i}\right)$ with $f_{A}\left(a^{i}\right) \in A^{i}$, otherwise $\varphi_{1}(g)=\varphi_{2}(g)=0$ if $f_{A}\left(a^{i}\right) \in A^{j}$ with $j \neq i$. Let $\theta_{i}$ be defined by

$$
\theta_{i}:=\gamma_{i}^{-1} i_{1}\left(w_{A}\right) f_{1}\left(\gamma_{i}\right) w_{1}^{-1}
$$

for each $i$ such that $f_{A}\left(a^{i}\right) \in A^{i}$ and otherwise $\theta_{i}:=1_{i}$. Because $\Lambda(A)$ is well-decomposed into $\bigoplus_{i=1}^{p} \Lambda\left(A^{i}\right)$ and for every $i$ we have

$$
\theta_{i} \varphi_{2}\left(\lambda_{i}\right)=\varphi_{1}\left(\lambda_{i}\right) \theta_{i}
$$

for each $\lambda_{i} \in \Lambda\left(A^{i}\right)$, according to proposition 2.1, there exists a well-defined group homomorphism $\theta_{*}: \mathcal{R}\left(f_{A}, w_{A}\right) \rightarrow \mathcal{R}\left(f_{1}, w_{1}\right)$. We will denote it by $i_{1 *}$. It turns out that

$$
i_{1 *}([g])=\left[\gamma_{i}^{-1} i_{1}(g) i_{1}\left(w_{A}\right) f_{1}\left(\gamma_{i}\right) w_{1}^{-1}\right]
$$

if $g \in \pi_{1}\left(A^{i}, a^{i}\right)$ with $f_{A}\left(a^{i}\right) \in A^{i}$. It is easy to see that it does not depend on the choice of the paths $\gamma_{i}$ in the sense that if other paths $\delta_{i}$ are chosen then the corresponding induced homomorphism is the same. We could do the same thing for $i_{2}, j_{1}$ and $j_{2}$, and it can be easily shown that $j_{2 *} i_{2 *}=j_{1 *} i_{1 *}$.

In other words the following diagram is commutative:

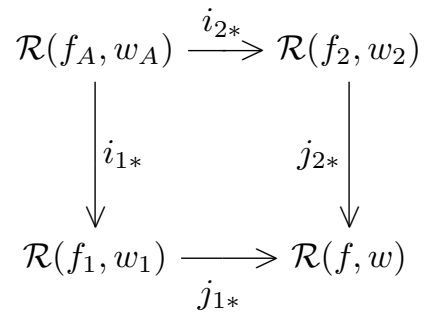

4. The pushout formula. We are now in a position to state the main theorem of this paper. If all the spaces involved are connected then the statement is the same as that of $[\mathrm{F}]$.

Theorem 4.1 (Pushout formula). Let $i_{1}: A \rightarrow X_{1}, i_{2}: A \rightarrow X_{2}, f_{A}: A \rightarrow A$, $f_{1}: X_{1} \rightarrow X_{1}$ and $f_{2}: X_{2} \rightarrow X_{2}$ be cellular maps such that $f_{1} i_{1}=i_{1} f_{A}$ and $f_{2} i_{2}=i_{2} f_{A}$. Let $f:=f_{1} \sqcup_{f_{A}} f_{2}$ be the pushout map of $f_{1}$ and $f_{2}$ via $f_{A}$. If $i_{1}$ is an inclusion, then

$$
\mathcal{L}(f, w)=j_{1 *} \mathcal{L}\left(f_{1}, w_{1}\right)+j_{2 *} \mathcal{L}\left(f_{2}, w_{2}\right)-j_{1 *} i_{1 *} \mathcal{L}\left(f_{A}, w_{A}\right) .
$$

Proof. Let $M\left(i_{2}\right)$ be the mapping cylinder of $i_{2}$ as defined in example 3.2. Let $i i_{2}: A \rightarrow M\left(i_{2}\right)$ be defined by $i i_{2}(a):=\overline{1}_{2}(a, 1)$ where $\overline{1}_{2}: A \times I \rightarrow M\left(i_{2}\right)$ is the map of the pushout construction, and let $p: M\left(i_{2}\right) \rightarrow X_{2}$ be defined by $p \overline{\mathrm{p}}_{2}(a, t)=i_{2}(a)$ for every $(a, t) \in A \times I$ and $p \overline{1}_{0}=1_{X_{2}}$. Well-known facts are that $i i_{2}$ is a cellular inclusion (hence a cofibration) and that $p$ is a homotopy equivalence whose inverse is $\overline{1}_{0}$. For more details see e.g. $[\mathrm{P}]$.

Let $f_{A \times I}: A \times I \rightarrow A \times I$ be defined by $f_{A \times I}(a, t)=\left(f_{A}(a), t\right)$ for all $(a, t) \in A \times I$. Then $f_{A \times I} i_{0}=i_{0} f_{A}$ and $f_{2} i_{2}=i_{2} f_{A}$, hence the pushout map $f_{A \times I} \sqcup_{f_{A}} f_{2}: M\left(i_{2}\right) \rightarrow$ $M\left(i_{2}\right)$ is defined. Denote it by $f_{2}^{\prime}$. It is a cellular self-map of $M\left(i_{2}\right)$ and $f_{2}^{\prime} i i_{2}=i i_{2} f_{A}$. Therefore the pushout map $f_{1} \sqcup_{f_{A}} f_{2}^{\prime}$ can be defined on the pushout space $X_{1} \sqcup_{A} M\left(i_{2}\right)$ of $i_{1}: A \rightarrow X_{1}$ and $i i_{2}: A \rightarrow M\left(i_{2}\right)$. Let $\bar{p}: X_{1} \sqcup_{A} M\left(i_{2}\right) \rightarrow X_{1} \sqcup A X_{2}$ be the cellular map such that $\bar{p} \bar{i} i_{2}=j_{1}$ and $\bar{p}_{1}=j_{2} p$. 
As we did in the previous section, induced homomorphisms are defined such that the following diagram commutes:

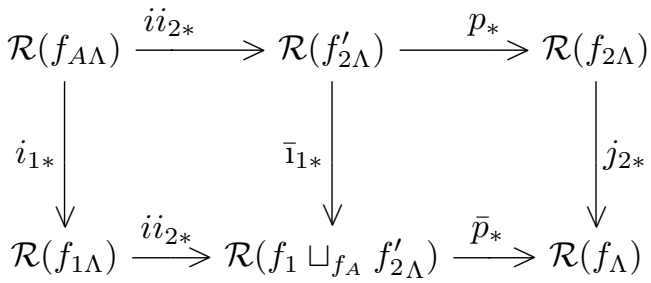

Let us note that base multipaths are omitted for the sake of simplicity.

LEMMA 4.2. We have the identities

$$
p_{*}\left(\mathcal{L}\left(f_{2}^{\prime}\right)\right)=\mathcal{L}\left(f_{2}\right), \quad \bar{p}_{*}\left(\mathcal{L}\left(f_{1} \sqcup_{f_{A}} f_{2}^{\prime}\right)\right)=\mathcal{L}(f) .
$$

PROOF. If all the spaces involved are connected this is exactly the statement of lemma 4.2 and lemma 4.3 of [F]. Otherwise $X_{2}$ or $X$ may be disconnected. But in this case, as $p$ and $\bar{p}$ are homotopy equivalences (see e.g. $[\mathrm{P}]$ ), they induce bijections at the 0 -homotopy set level $\pi_{0}\left(M\left(i_{2}\right)\right)=\pi_{0}\left(X_{2}\right)$ and $\pi_{0}\left(X_{1} \sqcup_{A} M\left(i_{2}\right)\right)=\pi_{0}(X)$. Moreover, according to proposition 3.2 ,

$$
\begin{array}{cc}
\mathcal{R}\left(f_{2 \Lambda}^{\prime}\right)=\bigoplus_{i=1}^{p_{0}^{\prime}} \mathcal{R}\left(f_{2}^{\prime}{ }_{\Lambda}\left(M\left(i_{2}\right)^{i}\right)\right), & \mathcal{L}\left(f_{2}^{\prime}\right)=\sum_{i=1}^{p_{0}^{\prime}} \mathcal{L}\left(f_{2}^{\prime}{ }^{\prime}\right), \\
\mathcal{R}\left(f_{2 \Lambda}\right)=\bigoplus_{i=1}^{p_{0}} \mathcal{R}\left(f_{2 \Lambda}^{i}\left(X_{2}^{i}\right)\right), \quad \mathcal{L}\left(f_{2}\right)=\sum_{i=1}^{p_{0}} \mathcal{L}\left(f_{2}^{i}\right),
\end{array}
$$

where $X_{2}^{1}, \ldots, X_{2}^{p_{0}}$ are the connected components of $X_{2}$ such that $f_{2}\left(X_{2}^{i}\right) \subset X_{2}^{i}$ and the same holds for $M\left(i_{2}\right)$. It can be seen that $p_{0}=p_{0}^{\prime}$ and that, as proved in $[\mathrm{F}]$,

$$
p_{*} \mathcal{L}\left(f_{2}^{\prime} i\right)=\mathcal{L}\left(f_{2}^{i}\right)
$$

because $f_{2}^{i}$ and $f_{2}^{i^{\prime}}$ are self-maps of connected spaces. Therefore, because of the additivity of $p_{*}$,

$$
p_{*}\left(\mathcal{L}\left(f_{2}^{\prime}\right)\right)=\mathcal{L}\left(f_{2}\right)
$$

and hence the first part of the lemma. The second one can be proved in the same way.

Because $\bar{p}_{*} \overline{i i}_{2 *}=j_{1 *}$ and $p_{*} i i_{2 *}=i_{2 *}$, we have

$$
j_{1 *} \mathcal{L}\left(f_{1}\right)+j_{2 *} \mathcal{L}\left(f_{2}\right)-j_{1 *} i_{1 *} \mathcal{L}\left(f_{A}\right)=\bar{p}_{*} \bar{i}_{2 *} \mathcal{L}\left(f_{1}\right)+\bar{p}_{*} \overline{1}_{1 *} \mathcal{L}\left(f_{2}^{\prime}\right)-\bar{p}_{*} \bar{i}_{2 *} i_{1 *} \mathcal{L}\left(f_{A}\right)
$$

and hence the pushout formula holds if and only if

$$
\mathcal{L}\left(f_{1} \sqcup_{f_{A}} f_{2}^{\prime}\right)=\overline{i i}_{2 *} \mathcal{L}\left(f_{1}\right)+\overline{1}_{1 *} \mathcal{L}\left(f_{2}^{\prime}\right)-\overline{i i}_{2 *} i_{1 *} \mathcal{L}\left(f_{A}\right) .
$$

Let us remark that both $i_{1}$ and $i i_{2}$ are supposed to be inclusions. This means that it suffices to prove the theorem in case both $i_{1}$ and $i_{2}$ are cellular inclusions.

Hence let us suppose that $A$ is a subcomplex of $X_{1}$ and $X_{2}$ and that $X=X_{1} \cup X_{2}$, $A=X_{1} \cap X_{2}$. Clearly $i_{1}: A \rightarrow X_{1}, i_{2}: A \rightarrow X_{2}, j_{1}: X_{1} \rightarrow X$ and $j_{2}: X_{2} \rightarrow$ $X$ are all inclusions. The maps $f_{A}, f_{1}$ and $f_{2}$ are simply the restrictions of $f$ to the subcomplexes $A, X_{1}$ and $X_{2}$. Let $w_{A}=w_{A}^{1}, w_{A}^{2}, \ldots, w_{1}^{1}, w_{1}^{2}, \ldots, w_{2}^{1}, w_{2}^{2}, \ldots$ and $w^{1}, w^{2}, \ldots$ be the base multipaths. Let $A^{1}, A^{2}, \ldots, A^{p_{A}}$ be the connected components of $A$ with base 
points $a^{1}, a^{2}, \ldots, a^{p_{A}}$; let $X_{1}^{1}, X_{1}^{2}, \ldots, X_{1}^{p_{1}}$ be those of $X_{1}$ with base points $x_{1}^{1}, x_{1}^{2}, \ldots, x_{1}^{p_{1}}$; $X_{2}^{1}, X_{2}^{2}, \ldots, X_{2}^{p_{2}}$, those of $X_{2}$ with base points $x_{2}^{1}, x_{2}^{2}, \ldots, x_{2}^{p_{2}}$ and $X^{1}, X^{2}, \ldots, X^{p}$ those of $X$ with base points $x^{1}, x^{2}, \ldots, x^{p}$. Let $\tilde{A}, \tilde{X}_{1}, \tilde{X}_{2}$ and $\tilde{X}$ be the universal covering spaces of $A, X_{1}, X_{2}$ and $X$, and $C_{q}(\tilde{A}), C_{q}\left(\tilde{X}_{1}\right), C_{q}\left(\tilde{X}_{2}\right)$ and $C_{q}(\tilde{X})$ their $q$-dimensional cellular chain groups. Fix an integer $q \geq 0$. Let $P_{A}: C_{q}(\tilde{A}) \rightarrow C_{q}(\tilde{A}), P_{1}: C_{q}\left(\tilde{X}_{1}\right) \rightarrow C_{q}\left(\tilde{X}_{1}\right)$, $P_{2}: C_{q}\left(\tilde{X}_{2}\right) \rightarrow C_{q}\left(\tilde{X}_{2}\right)$ and $P: C_{q}(\tilde{X}) \rightarrow C_{q}(\tilde{X})$ be the projection homomorphisms as defined in section 3.1. Now let us consider the canonical liftings of $\left(f_{A}, w_{A}\right),\left(f_{1}, w_{1}\right)$, $\left(f_{2}, w_{2}\right)$ and $(f, w)$ and the corresponding chain homomorphisms

$$
C_{q}\left(\tilde{f}_{A}\right), C_{q}\left(\tilde{f}_{1}\right), C_{q}\left(\tilde{f}_{2}\right), C_{q}(\tilde{f})
$$

at the $q$-dimensional chain group level. Then the theorem follows easily from the following lemma.

Lemma 4.3. For any $q \geq 0$ we have the identity

$$
\begin{aligned}
j_{1 *}\left(\operatorname{Tr}_{f_{1 \Lambda}}\left(C_{q}\left(\tilde{f}_{1}\right) P_{1}\right)\right)+j_{2 *}( & \left.\operatorname{Tr}_{f_{2 \Lambda}}\left(C_{q}\left(\tilde{f}_{2}\right) P_{2}\right)\right) \\
& -j_{1 *} i_{1 *}\left(\operatorname{Tr}_{f_{A \Lambda}}\left(C_{q}\left(\tilde{f}_{A}\right) P_{A}\right)\right)=\operatorname{Tr}_{f_{\Lambda}}\left(C_{q}(\tilde{f}) P\right) .
\end{aligned}
$$

Proof. Let us start by considering the square diagram

$$
\begin{gathered}
C_{q}(\tilde{A}) \leftarrow \frac{C_{q}\left(\tilde{f}_{A}\right) P_{A}}{\longleftarrow} C_{q}(\tilde{A}) \\
C_{q}\left(\tilde{i}_{1}^{\prime}\right) P_{A} \quad C_{q}\left(\tilde{i}_{1}\right) P_{A} \\
\downarrow \\
C_{q}\left(\tilde{X}_{1}\right) \longleftarrow
\end{gathered}
$$

where $C_{q}\left(\tilde{f}_{A}\right), C_{q}\left(\tilde{f}_{1}\right)$ and $P_{A}$ are defined as above, and $\tilde{i}_{1}$ and $\tilde{i}_{1}^{\prime}$ are defined as follows: for each $j=1, \ldots, p_{A}$ let $\gamma_{j}$ be a continuous path $\gamma_{j}:(I, 0,1) \rightarrow\left(X_{1}, a^{j}, x_{1}^{j^{\prime}}\right)$ where $a^{j}$ is the base point of $A^{j}$ and $x_{1}^{j^{\prime}}$ is the base point of the component $X_{1}^{j^{\prime}}$ of $X_{1}$ which contains $A^{j}$; let us remark that points of $\tilde{A}$ are homotopy classes rel. endpoints of paths $\lambda:(I, 0) \rightarrow\left(A,\left\{a^{1}, a^{2}, \ldots, a^{p_{A}}\right\}\right)$ and points of $\tilde{X}_{1}$ are homotopy classes of paths $\lambda:$ $(I, 0) \rightarrow\left(X_{1},\left\{x_{1}^{1}, x_{1}^{2}, \ldots, x_{1}^{p_{1}}\right\}\right)$; we set

$$
\tilde{i}_{1}([\lambda]):=\left[\gamma_{j}^{-1} \lambda\right]
$$

for each $\lambda:(I, 0) \rightarrow\left(A^{j}, a^{j}\right)$ and

$$
\tilde{i}_{1}^{\prime}([\lambda]):= \begin{cases}{\left[w_{1}^{j} f_{1}\left(\gamma_{j}^{-1}\right)\left(w_{A}^{j}\right)^{-1} \lambda\right]} & \text { if } f_{A}\left(a^{j}\right) \in A^{j} \\ {\left[\gamma_{j}^{-1} \lambda\right]} & \text { otherwise }\end{cases}
$$

for each $\lambda:(I, 0) \rightarrow\left(A^{j}, a^{j}\right)$.

It is not difficult to see that the diagram is commutative. Moreover, if we recall the definition of $i_{1 *}$ of section 3.2 by taking $\varphi_{1}, \varphi_{1}^{\prime}: \Lambda(A) \rightarrow \Lambda\left(X_{1}\right)$ as the unique ring homomorphisms such that

$$
\varphi_{1}(g):= \begin{cases}\gamma_{j}^{-1} g \gamma_{j} & \left(\forall g \in \pi_{1}\left(A^{j}, a^{j}\right) \text { s.t. } f\left(a^{j}\right) \in A^{j}\right) \\ 0 & \text { otherwise }\end{cases}
$$




$$
\varphi_{1}^{\prime}(g):= \begin{cases}w_{1}^{j} f_{1}\left(\gamma_{j}^{-1}\right)\left(w_{A}^{j}\right)^{-1} g w_{A}^{j} f_{1}\left(\gamma_{j}\right) w_{1}^{j-1} & \left(\forall g \in \pi_{1}\left(A^{j}, a^{j}\right),\right. \\ 0 & \text { s.t. } \left.f\left(a^{j}\right) \in A^{j}\right), \\ \text { otherwise }\end{cases}
$$

we get the commutative diagram

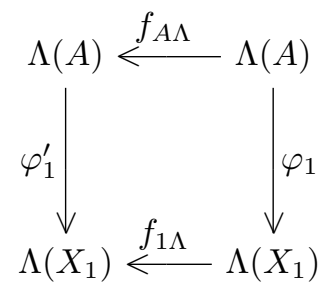

of ring homomorphisms. The main point is that $C_{q}\left(\tilde{i}_{1}\right) P_{A}$ is a $\varphi_{1}$-homomorphism and $C_{q}\left(\tilde{i}_{1}^{\prime}\right) P_{A}$ is a $\varphi_{1}^{\prime}$-homomorphism. Moreover, as shown in section $3.2, i_{1 *}: \mathcal{R}\left(f_{A}, w_{A}\right) \rightarrow$ $\mathcal{R}\left(f_{1}, w_{1}\right)$ is defined by

$$
i_{1 *}([\lambda]):=\left[\theta_{j} \varphi_{1}^{\prime}(\lambda)\right]\left(\forall \lambda \in \Lambda\left(A^{j}\right) \text { s.t. } f\left(a^{j} \in A^{j}\right)\right)
$$

and is zero otherwise, where $\theta_{j}$ is a suitable element of $\Lambda\left(X_{1}\right)$ such that $\theta_{j} \varphi_{1}^{\prime}(\lambda)=\varphi_{1}(\lambda) \theta_{j}$ for each $\lambda \in \Lambda\left(A^{j}\right)$.

The same arguments apply to $i_{2}, j_{1}$ and $j_{2}$; therefore we get the following commutative diagram of ring homomorphisms:

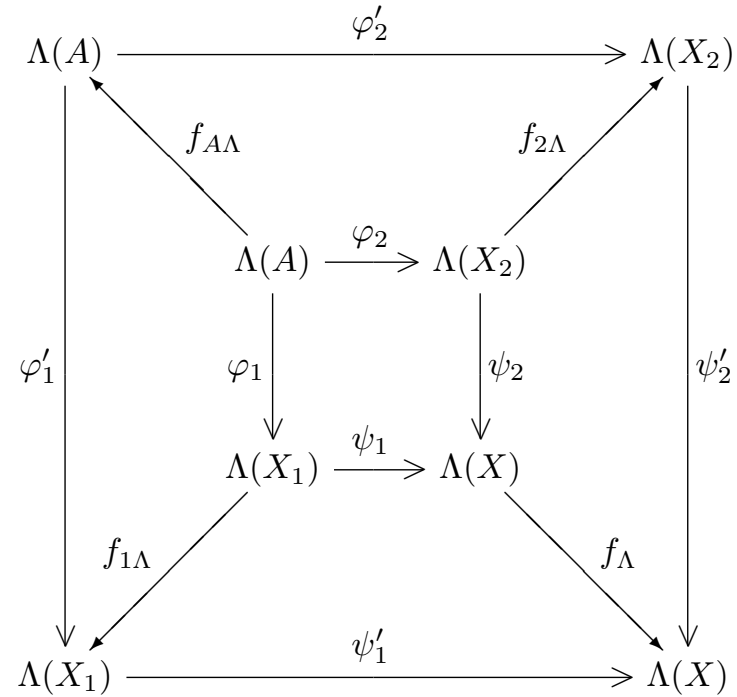

which induces, at the Reidemeister group level, the diagram of section 3.2.

Now, let $Q_{A}$ be a $\Lambda(A)$-module such that $C_{q}(\tilde{A}) \oplus Q_{A}$ is a free finitely generated $\Lambda(A)$-module. As shown in the proof of proposition 3.1 and in $[\mathrm{H}],[\mathrm{FH}]$ we can suppose that a free basis of $C_{q}(\tilde{A}) \oplus Q_{A}$ can be given by taking liftings of the $q$-dimensional cells of $A\left\{e_{1}, e_{2}, \ldots, e_{k}\right\}$ to $\tilde{A}$ (and hence they can be thought of as elements of $C_{q}(\tilde{A})$ ). If we consider the $f_{A \Lambda}$-endomorphism $C_{q}\left(f_{A}\right) P+0_{Q_{A}}$ of $C_{q}(\tilde{A}) \oplus Q_{A}$ we get exactly the $f_{A \Lambda}$-endomorphism whose trace is the trace of $C_{q}\left(\tilde{f}_{A}\right) P_{A}$. The same argument applies to $X_{1}, X_{2}$ and $X$, and therefore we have the $f_{1 \Lambda}$-endomorphism $C_{q}\left(\tilde{f}_{1}\right) P_{1}+0_{Q_{1}}$ of the free 
finitely generated $\Lambda\left(X_{1}\right)$-module $C_{q}\left(\tilde{X}_{1}\right) \oplus Q_{1}$, the $f_{2 \Lambda^{-}}$-endomorphism $C_{q}\left(\tilde{f}_{2}\right) P_{2}+0_{Q_{2}}$ of the free finitely generated $\Lambda\left(X_{2}\right)$-module $C_{q}\left(\tilde{X}_{2}\right) \oplus Q_{2}$ and the $f_{\Lambda}$-endomorphism $C_{q}(\tilde{f}) P+0_{Q}$ of the free finitely generated $\Lambda(X)$-module $C_{q}(\tilde{X}) \oplus Q$. Their traces are by definition exactly the traces of the corresponding endomorphisms at the chain group level. Let us call them $F_{A}, F_{1}, F_{2}$ and $F$.

Finally we define the homomorphisms corresponding to $i_{1}, i_{2}, j_{1}$ and $j_{2}$. As an example, let $\Phi_{1}:=C_{q}\left(\tilde{i_{1}}\right) P_{A}+0_{Q_{A}}: C_{q}(\tilde{A}) \oplus Q_{A} \rightarrow C_{q}(\tilde{A}) \oplus Q_{A}$ be defined in the obvious way. The others are defined in the same way. We arrange them as in the following diagram.



Let us note that

$$
\left(\forall \lambda \in \Lambda\left(A^{j}\right)\right) \Phi_{1}^{\prime}(\lambda)=\Phi_{1}(\lambda) \cdot \theta_{j} .
$$

Similarly $\left(\forall j=1, \ldots, p_{A}\right)$ there exist elements $\eta_{j} \in \Lambda\left(X_{2}\right)$ such that

$$
\left(\forall \lambda \in \Lambda\left(A^{j}\right)\right) \Phi_{2}^{\prime}(\lambda)=\Phi_{2}(\lambda) \cdot \eta_{j}
$$

and $\left(\forall j=1, \ldots, p_{1}, \forall i=1, \ldots, p_{2}\right)$ there exist $\zeta_{j}, \epsilon_{i} \in \Lambda(X)$ such that

$$
\left(\forall \lambda \in \Lambda\left(X_{1}^{j}\right)\right) \Phi_{1}^{\prime}(\lambda)=\Phi_{1}(\lambda) \cdot \zeta_{j}
$$

and

$$
\left(\forall \lambda \in \Lambda\left(X_{2}^{i}\right)\right) \Phi_{2}^{\prime}(\lambda)=\Phi_{2}(\lambda) \cdot \epsilon_{i}
$$

for each $j=1, \ldots, p_{1}$ and $i=1, \ldots, p_{2}$. Moreover as before $i_{1 *}=\theta_{*}, i_{2 *}=\eta_{*}, j_{1 *}=\epsilon_{*}$ and $j_{2 *}=\zeta_{*}$. Let us recall that these homomorphisms do not depend on the choice of the paths that occur in the definition of $\theta_{j}, \eta_{j}, \zeta_{j}$ and $\epsilon_{j}$.

As shown in $[\mathrm{H}],[\mathrm{F}]$ we can take a free $\Lambda\left(X_{1}\right)$-basis $b_{1}, b_{2}, \ldots, b_{k+s}$ of $C_{q}\left(\tilde{X}_{1}\right) \oplus Q_{1}$ such that $b_{j}=\Phi_{1}\left(e_{j}\right)$ for each $j=1, \ldots, k$ such that $e_{j}$ and $f_{A}\left(e_{j}\right)$ belong to the same connected component of $A$. In the same way let $c_{1}, c_{2}, \ldots, c_{k+t}$ be a free $\Lambda\left(X_{2}\right)$-basis of $C_{q}\left(\tilde{X}_{2}\right) \oplus Q_{2}$ such that $c_{j}=\Phi_{2}\left(e_{j}\right)$ for each $j=1, \ldots, k$ such that $e_{j}$ and $f_{A}\left(e_{j}\right)$ belong to the same connected component of $A$. Therefore we can take a free $\Lambda(X)$-basis $d_{1}, d_{2}, \ldots, d_{k+s+t}$ of $C_{q}(\tilde{X}) \oplus Q$ such that $d_{j}=\Psi_{1}\left(b_{j}\right)$ for all $j=1, \ldots, k+s$ such that 
$f_{1}\left(b_{j}\right)$ and $b_{j}$ are in the same connected component of $X_{1}$, and $d_{j}=\Psi_{2}\left(c_{j-s}\right)$ for all $j=$ $k+s+1, \ldots, k+s+t$ such that $f_{2}\left(c_{j}\right)$ and $c_{j}$ are in the same connected component of $X_{2}$.

Let $E_{v u}, B_{v u}, C_{v u}$ and $D_{v u}$ be the entries of the matrices representing $F_{A}, F_{1}, F_{2}$ and $F$ respectively. In other words $\left\{E_{v u}\right\} \in \mathcal{M}_{k, k}(\Lambda(A))$ and

$$
F_{A}\left(e_{u}\right)=\sum_{v=1}^{k} e_{v} E_{v u}
$$

for all $u=1, \ldots, k ;\left\{B_{v u}\right\} \in \mathcal{M}_{k+s, k+s}\left(\Lambda\left(X_{1}\right)\right)$ and

$$
F_{1}\left(b_{u}\right)=\sum_{v=1}^{k+s} b_{v} B_{v u}
$$

for all $u=1, \ldots, k+s ;\left\{C_{v u}\right\} \in \mathcal{M}_{k+t, k+t}\left(\Lambda\left(X_{2}\right)\right)$ and

$$
F_{2}\left(c_{u}\right)=\sum_{v=1}^{k+t} c_{v} C_{v u}
$$

for all $u=1, \ldots, k+t$; finally $\left\{D_{v u}\right\} \in \mathcal{M}_{k+s+t, k+s+t}(\Lambda(X))$ and

$$
F\left(d_{u}\right)=\sum_{v=1}^{k+s+t} d_{v} D_{v u}
$$

for all $u=1, \ldots, k+s+t$.

We know that

$$
\operatorname{Tr}_{f_{\Lambda}}\left(C_{q}(\tilde{f}) P\right)=\operatorname{Tr}_{f_{\Lambda}}(F)=\sum_{u=1}^{k+s+t}\left[D_{u u}\right]
$$

and that similar formulae hold for $C_{q}\left(\tilde{f}_{A}\right) P_{A}, C_{q}\left(\tilde{f}_{1}\right) P_{1}$ and $C_{q}\left(\tilde{f}_{2}\right) P_{2}$. But for $u=$ $1, \ldots, k+s$

$$
F\left(d_{u}\right)=F\left(\Psi_{1}\left(b_{u}\right)\right)=\Psi_{1}^{\prime} F_{1}\left(b_{u}\right)
$$

and hence

$$
F\left(d_{u}\right)=\sum_{u=1}^{k+s} \Psi_{1}^{\prime}\left(b_{v} B_{v u}\right)
$$

for each $u=1, \ldots, k+s$. Because $\Psi_{1}^{\prime}$ is a $\psi_{1}^{\prime}$-homomorphism, $\Psi_{1}^{\prime}\left(b_{v} B_{v u}\right)=\Psi_{1}^{\prime}\left(b_{v}\right) \psi_{1}^{\prime}\left(B_{v u}\right)$ for all $u, v=1, \ldots, k+s$. But for a suitable $j$ depending on $v, \Psi_{1}^{\prime}\left(b_{v}\right)=\Psi_{1}\left(b_{v}\right) \zeta_{j(v)}$ and hence $\Psi_{1}^{\prime}\left(b_{v}\right)=d_{v} \zeta_{j(v)}$. This implies that

$$
F\left(d_{u}\right)=\sum_{u=1}^{k+s} d_{v}\left(\zeta_{j(v)} \psi_{1}^{\prime}\left(B_{v u}\right)\right)
$$

for all $u=1, \ldots, k+s$ and hence that

$$
D_{u}^{u}=\zeta_{j(u)} \psi_{1}^{\prime}\left(B_{u u}\right)
$$

for $u=1, \ldots, k+s$. In a similar way we can show that

$$
D_{u u}=\epsilon_{j^{\prime}(u-s)} \psi_{2}^{\prime}\left(C_{u-s, u-s}\right)
$$

for all $u=k+s+1, \ldots, k+s+t$ and suitable indices $j^{\prime}$ depending on $u$; moreover for other $i$ 's depending on $u$

$$
B_{u u}=\theta_{i(u)} \phi_{1}^{\prime}\left(E_{u u}\right), \quad C_{u u}=\eta_{i(u)} \phi_{2}^{\prime}\left(E_{u u}\right)
$$

for all $u=1, \ldots, k$. 
Now by definition of $i_{1 *}, i_{2 *}, j_{1 *}$ and $j_{2 *}$

$$
\begin{aligned}
j_{1 *}\left(\operatorname{Tr}_{f_{1 \Lambda}}\left(C_{q}\left(\tilde{f}_{1}\right) P_{1}\right)\right) & =\sum_{u=1}^{k+s}\left[\zeta_{j(u)} \psi_{1}^{\prime}\left(B_{u u}\right)\right] \\
j_{2 *}\left(\operatorname{Tr}_{f_{2 \Lambda}}\left(C_{q}\left(\tilde{f}_{2}\right) P_{2}\right)\right) & =\sum_{u=1}^{k+t}\left[\epsilon_{j^{\prime}(u)} \psi_{2}^{\prime}\left(C_{u u}\right)\right] \\
j_{1 *} i_{1 *}\left(\operatorname{Tr}_{f_{A \Lambda}}\left(C_{q}\left(\tilde{f}_{A}\right) P_{A}\right)\right) & =\sum_{u=1}^{k}\left[\epsilon_{j^{\prime}(u)} \psi_{2}^{\prime}\left(\eta_{i(u)} \phi_{2}^{\prime}\left(E_{u u}\right)\right)\right]
\end{aligned}
$$

and hence

$$
\begin{aligned}
j_{1 *}\left(\operatorname{Tr}_{f_{1 \Lambda}}\left(C_{q}\left(\tilde{f}_{1}\right) P_{1}\right)\right)+j_{2 *}\left(\operatorname{Tr}_{f_{2 \Lambda}}(\right. & \left.\left.C_{q}\left(\tilde{f}_{2}\right) P_{2}\right)\right) \\
= & \operatorname{Tr}_{f_{\Lambda}}\left(C_{q}(\tilde{f}) P\right)+\sum_{u=1}^{k}\left[\epsilon_{j^{\prime}(u)} \psi_{2}^{\prime}\left(C_{u u}\right)\right] \\
= & \operatorname{Tr}_{f_{\Lambda}}\left(C_{q}(\tilde{f}) P\right)+j_{1 *} i_{1 *}\left(\operatorname{Tr}_{f_{A \Lambda}}\left(C_{q}\left(\tilde{f}_{A}\right) P_{A}\right)\right)
\end{aligned}
$$

concluding the proof.

With this lemma the proof of the theorem is complete. Let us note that the hypothesis that at least $i_{1}$ is an inclusion cannot be omitted. Let $A=S^{1}$ be a circle and let $X_{1}=$ $X_{2}=\{*\}$ be a single point. Then $i_{1}: A \rightarrow X_{1}$ and $i_{2}: A \rightarrow X_{2}$ are constant maps, and the pushout space $X=X_{1} \sqcup_{A} X_{2}$ is a single point $\{*\}$ too. Therefore if $f_{A}: S^{1} \rightarrow S^{1}$ is a map of degree $d$ and $f_{1}$ and $f_{2}$ are constant maps, then the pushout map $f$ is the obvious constant map, and $\mathcal{L}(f)=[1] \neq \mathcal{L}\left(f_{1}\right)+\mathcal{L}\left(f_{2}\right)-\mathcal{L}\left(f_{A}\right)$ because the right hand side of this equation is equal to [1] $+[1]-(1-d)[1]=(d+1)[1] \neq[1]$.

\section{References}

[B] R. F. Brown, The Lefschetz Fixed Point Theorem, Scott Foresman and Company, Chicago, 1971.

[FH] E. FAdell and S. Husseini, The Nielsen Number on Surfaces, Contemp. Math. 21, AMS, Providence, 1983.

[F] D. Ferrario, Generalized Lefschetz numbers of pushout maps, Topology Appl. 68 (1996) $67-81$.

[H] S. Y. Husseini, Generalized Lefschetz Numbers, Trans. Amer. Math. Soc. 272 (1982), 247-274.

[J] B. J. JiAng, Lectures on Nielsen fixed point theory, Contemp. Math. 14, Amer. Math. Soc., Providence, 1983.

[J1] B. J. JIAng, Periodic orbits on surfaces via Nielsen fixed point theory, in: Topology Hawaii (Honolulu, HI, 1990), 101-118.

[P] R. A. Piccinini, Lectures on Homotopy Theory, North-Holland, Amsterdam, 1992.

[S] J. Stallings, Centerless groups - an algebraic formulation of Gottlieb's theorem, Topology 4 (1965), 129-134. 